\title{
Prethermal memory loss in interacting quantum systems coupled to thermal baths
}

\author{
Ling-Na $\mathrm{Wu}^{*}$ and André Eckardt ${ }^{\dagger}{ }^{\dagger}$ \\ Technische Universität Berlin, Institut für Theoretische Physik, Hardenbergstrasse 36, D-10623 Berlin, Germany \\ and Max Planck Institute for the Physics of Complex Systems, Nöthnitzer Strasse 38, D-01187 Dresden, Germany
}

(Received 28 November 2019; revised manuscript received 3 June 2020; accepted 3 June 2020; published 18 June 2020)

\begin{abstract}
We study the relaxation dynamics of an extended Fermi-Hubbard chain with a strong Wannier-Stark potential tilt coupled to a bath. When the system is subjected to dephasing noise, starting from a pure initial state the system's total von Neumann entropy is found to grow monotonously. The scenario becomes rather different when the system is coupled to a thermal bath of finite temperature. Here, for sufficiently large field gradients and initial energies, the entropy peaks in time and almost reaches its largest possible value (corresponding to the maximally mixed state), long before the system relaxes to thermal equilibrium. This entropy peak signals an effective prethermal memory loss and, relative to the time where it occurs, the system is found to exhibit a simple scaling behavior in space and time. By comparing the system's dynamics to that of a simplified model, the underlying mechanism is found to be related to the localization property of the Wannier-Stark system, which favors dissipative coupling between eigenstates that are close in energy.
\end{abstract}

DOI: 10.1103/PhysRevB.101.220302

Introduction. The problem of particles moving in a tilted lattice (Wannier-Stark system) is associated with various interesting phenomena, such as Bloch oscillations [1], Stark localization [2], and Landau-Zener tunneling [3]. Despite its long history, it has remained a frontier research topic both theoretically [4] and experimentally [5-16]. Recent studies $[17,18]$ show that an interacting Wannier-Stark system can exhibit nonergodic behavior analogous to disorder-induced many-body localization (MBL) [19-22]. Understanding both the differences and similarities between such disorder-free and conventional MBL constitutes a fundamental question, which currently attracts a lot of attention [23-36].

On the other hand, there is increased recent interest in the nonequilibrium properties of open many-body quantum systems [37-69]. While the coupling to an environment of finite temperature constitutes a natural situation, it is rather cumbersome to simulate. Therefore, the impact of dissipation is often treated by using dephasing noise [70] as a simpler bath model. Even though dephasing noise will eventually drive the system into an infinite-temperature state, it is assumed to qualitatively capture the effect of weak coupling to a bath on the transient evolution. Understanding under which circumstances this assumption breaks down is an important question for the simulation of open many-body systems (see also Refs. [71,72]).

\footnotetext{
*lingna.wu@tu-berlin.de

†eckardt@tu-berlin.de
}

Published by the American Physical Society under the terms of the Creative Commons Attribution 4.0 International license. Further distribution of this work must maintain attribution to the author(s) and the published article's title, journal citation, and DOI. Open access publication funded by the Max Planck Society.
Here, we report on a surprising phenomenon in the relaxation dynamics of an interacting Wannier-Stark system coupled to a thermal bath of finite temperature. It sheds light on both of the above questions, since it can neither be observed for a disorder-localized system nor for dephasing noise. In a large parameter regime, we find that long before the system reaches thermal equilibrium, it transiently approaches the maximally mixed state. This effect implies an effective prethermal memory loss. It is reminiscent of the universal dynamics recently observed in isolated quantum gases [73,74].

Model. We consider a one-dimensional extended Hubbard chain half filled with spin-polarized fermions and subjected to a linear potential gradient. It is described by the Hamiltonian

$$
H=-J \sum_{i=1}^{M-1}\left(c_{i}^{\dagger} c_{i+1}+c_{i+1}^{\dagger} c_{i}\right)+\sum_{i=1}^{M} W_{i} n_{i}+V \sum_{i=1}^{M-1} n_{i} n_{i+1}
$$

Here, $c_{i}^{\dagger}$ and $n_{i}=c_{i}^{\dagger} c_{i}$ are the creation and number operators for a fermion on lattice site $i$. Moreover, $J$ is the tunneling parameter, $W_{i}=-r i$ captures the potential gradient $r$, and $V$ quantifies nearest-neighbor interactions. This model is equivalent to an XXZ chain via a Jordan-Wigner transform [75]. The single-particle (bulk) eigenstates, known as Wannier-Stark states, are centered at the lattice sites, with a localization length $\sim J / r$ (with respect to the site index $i$ ) and energies that increase by $r$ from site to site [2]. Moreover, also the interacting system shows properties akin to $\operatorname{MBL}[17,18]$. Henceforth, we use $J, J / k_{B}$, and $\hbar / J$ as units for energy, temperature, and time, respectively, so that $J=\hbar=k_{B}=1$.

When coupled weakly to a thermal bath, which is modeled as a collection of harmonic oscillators in thermal equilibrium and couples to the on-site occupations, the system can be 
described by a Redfield master equation [37],

$$
\begin{aligned}
\frac{d \rho}{d t}= & -i[H, \rho]+\eta \sum_{k, q, p, l=1}^{M}\left[R_{k q l p}\left(L_{k q} \rho L_{p l}^{\dagger}-L_{p l}^{\dagger} L_{k q} \rho\right)\right. \\
& \left.+R_{p l q k}\left(L_{k q} \rho L_{p l}^{\dagger}-\rho L_{p l}^{\dagger} L_{k q}\right)\right],
\end{aligned}
$$

with jump operators $L_{k q}=|k\rangle\langle q|$ between many-body eigenstates $|k\rangle$ of energy $\varepsilon_{k}[46,58]$. The corresponding transition rates read $R_{k q p l}=\pi v_{k q p l} g\left(\varepsilon_{k}-\varepsilon_{q}\right)$, with $v_{k q p l}=$ $\sum_{i=1}^{M}\left\langle k\left|n_{i}\right| q\right\rangle\left\langle p\left|n_{i}\right| l\right\rangle$ and bath correlation function $g(E)=$ $J(E) /\left(e^{E / T}-1\right)$, where we assume an Ohmic spectral density $J(E)=E$.

In the high-temperature limit, we have $g(E) \simeq T$ and the transition rate $R_{k q p l}$ becomes independent of energy. Thus, the master equation reduces to

$$
\frac{d \rho}{d t}=-i[H, \rho]+\gamma \sum_{i=1}^{M}\left(n_{i} \rho n_{i}-\frac{1}{2} n_{i}^{2} \rho-\frac{1}{2} \rho n_{i}^{2}\right),
$$

with $\gamma=\eta T$, describing dephasing noise.

Prethermal memory loss. Figure 1 shows the time evolution of the von Neumann entropy $S=-\operatorname{tr}\{\rho \log (\rho)\}$ of the total system, when coupled to a dephasing bath [Fig. 1(a)] or a finite-temperature bath [Fig. 1(b)]. It is calculated by numerically integrating Eqs. (3) and (2), respectively, starting from a Fock state with the left half of the chain occupied. The temperature $T$ of the thermal bath is chosen such that the corresponding equilibrium entropy $S_{T}$ (obtained for the Gibbs state $\rho_{T}=\mathcal{Z}_{T}^{-1} \sum_{k} e^{-\varepsilon_{k} / T}|k\rangle\langle k|$ with $\left.\mathcal{Z}_{T}=\sum_{k} e^{-\varepsilon_{k} / T}\right)$ is equal to half the largest possible entropy $S_{\infty} \equiv S_{T=\infty}=$ $\log (\mathcal{D})$, with Hilbert space dimension $\mathcal{D}=M ! /[(M / 2) !]^{2}$ (see Fig. S1 of Ref. [76] for other temperatures). For dephasing noise [Fig. 1(a)], the entropy grows monotonously to the maximum value $S_{\infty}$, being insensitive to the sign of the potential gradient $r$. In turn, when the system is coupled to the finite-temperature bath [Fig. 1(b)], the entropy approaches its equilibrium value rather differently for negative and positive $r$. While in the former case the entropy grows monotonously to $S_{T}$ (except for small $|r|$ ), in the latter case it first reaches a peak value $S_{\text {peak }}$ well above $S_{T}$, before relaxing to equilibrium. As will become apparent in the following, this difference can be attributed to the different mean energies of the initial states.

Remarkably, we can observe in Fig. 1(b) that for large positive gradients $r$, the peak entropy almost reaches the largest possible entropy $S_{\infty}$ (dotted line), which uniquely corresponds to the maximally mixed state $\rho_{\infty} \equiv \rho_{T=\infty}=$ $\mathcal{D}^{-1} \sum_{k}|k\rangle\langle k|$. This effect can be observed for a wide range of initial conditions: In Fig. 1(c) we plot the peak entropies $S_{\text {peak }}$ reached during the evolution starting from various initial Fock states, versus their mean energy $E_{\text {ini }}$ (scaled between 0 , for the ground-state energy $\varepsilon_{\min }$, and 1 , for the energy $\varepsilon_{\max }$ of the most excited state). Peak entropies close to $S_{\infty}$ are found as long as $E_{\text {ini }}$ lies well above the energy $E_{\infty}=\operatorname{tr}\left\{\rho_{\infty} H\right\}$ of the maximally mixed state (dashed line).

Since the maximally mixed state is unique, reaching an entropy peak with $S_{\text {peak }} \approx S_{\infty}$ indicates that we can expect the system dynamics to become (approximately) independent of the initial conditions near and after approaching the peak entropy. Such a behavior is confirmed in Fig. 1(d), where we (a)
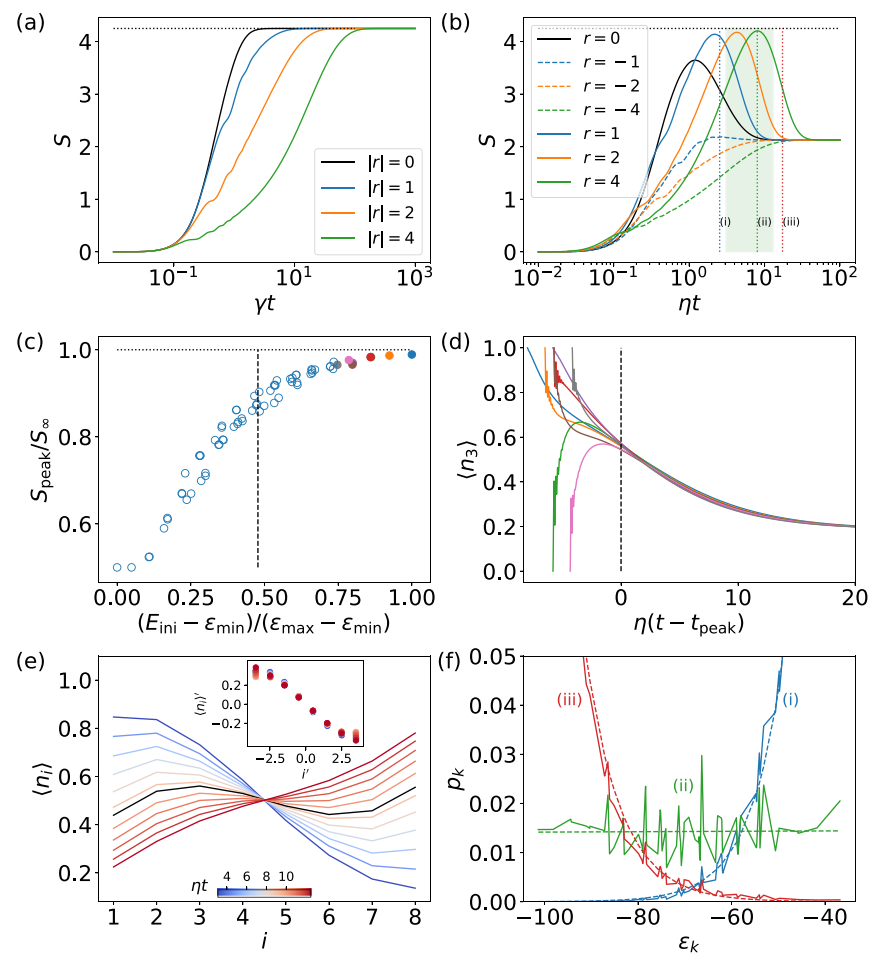

FIG. 1. (a), (b) Time evolution of the von Neumann entropy for the tilted ladder (1) initialized in a Fock state with the left half of the chain occupied and coupled to (a) a dephasing bath [Eq. (3)] or (b) a thermal bath [Eq. (2)]. The dotted line marks the largest possible entropy $S_{\infty}$. (c) Normalized peak entropy for initial Fock states of various energies $E_{\text {ini }}$. The vertical dashed line marks $E_{\infty}$. (d) Evolution of the mean occupation of the third lattice site relative to the time $t_{\text {peak }}$, where the entropy peak is reached. The line colors mark different initial Fock states corresponding to colored bullets in (c). (e) Density profile $\left\langle n_{i}\right\rangle$ at equidistant times during the time window marked by the shaded area in (b). The black line marks $t=$ $t_{\text {peak }}$. The inset shows the collapse of all the curves by rotating them by an angle proportional to the corresponding time. (f) Diagonal elements of the density matrix $p_{k}$ at three points in time [marked by (i)-(iii) in (b)] (solid lines) compared to effective thermal states of identical average energy (dashed lines). The parameters are $M=8$, $V=1, \gamma=\eta=0.1$, and $T$ so that $S_{T}=S_{\infty} / 2$. The field gradient for (c)-(f) is $r=4$.

plot the evolution of the site occupation $\left\langle n_{i=3}\right\rangle$ relative to the time $t_{\text {peak }}$ at which the entropy peak is reached. The different curves, which correspond to different initial states [labeled by line colors corresponding to the colored bullets in Fig. 1(c)], clearly converge near $\eta\left(t-t_{\text {peak }}\right)=0$ and subsequently show almost identical behavior. Similar behavior can also be observed for other site occupations $\left\langle n_{i}\right\rangle$ and for larger systems (see Figs. S3, S4 and Figs. S6, S9 of Ref. [76], respectively). Thus, the system undergoes an effective [77] prethermal memory loss, long before it reaches thermal equilibrium.

Moreover, we also find that the way the system approaches the maximally mixed state shows a simple form of scaling behavior. In Fig. 1(e) we plot the density distribution $\left\langle n_{i}\right\rangle$ at various times near $t_{\text {peak }}$ [within the shaded area in Fig. 1(b)], for $r=4$ and starting from the Fock state with the left half of the chain occupied [green curve in Fig. 1(b)]. These density 
profiles collapse on top of each other when rotated by an angle proportional to the evolved time (see the inset). Note that for $r=4$ the Wannier-Stark states are already well localized on single lattice sites, so that the plotted density profile (which can directly be measured in quantum-gas systems) approximately corresponds to the occupation of the single-particle eigenstates. In this sense, the observed behavior is somewhat reminiscent of the universal scaling behavior recently observed in the occupations of long-wavelength momentum modes during the far-from-equilibrium dynamics of isolated quantum gases [73,74], which was associated with the presence of nonthermal fixed points [78-80].

Further insight on how the system approaches the maximally mixed state is gained by looking at the probability distribution $p_{k}=\langle k|\rho| k\rangle$ for occupying many-body energy eigenstates $|k\rangle$. In Fig. 1(f) we plot the distribution $p_{k}$ (solid lines) for $r=4$ at three times: (i) slightly before, (ii) at, and (iii) slightly after $t_{\text {peak }}$ [as indicated in Fig. 1(b) relative to the green curve]. Interestingly, these distributions agree rather well with those for thermal states $\rho_{T_{\text {eff }}}$ (dashed lines) with the effective temperature $T_{\text {eff }}$ determined by the instantaneous energy, $\operatorname{tr}\left\{\rho_{T_{\mathrm{eff}}} H\right\} \equiv E=\operatorname{tr}\{\rho H\}$. This observation suggests that the prethermal memory loss is due to a dissipative form of prethermalization, where the system rapidly approaches a Gibbs state, whose effective temperature $T_{\text {eff }}$ then slowly relaxes to the equilibrium temperature $T$. This scenario immediately explains that an infinite-temperature state is approached long before the system has thermalized as long as the initial energy $E_{\text {ini }}$ lies well above the infinite-temperature energy $E_{\infty}$. Namely, in this case the system has enough time to approach a prethermal state $\rho_{T_{\text {eff }}}$, before $1 / T_{\text {eff }}$ passes through zero from below at the time when $E$ drops below $E_{\infty}$.

The prethermal relaxation to a Gibbs-like state with slowly varying effective temperature occurs in a way rather different from standard (pre)thermalization [81]. It cannot be understood as the prethermalization of the system and bath together, since the bath always remains in the same thermal state with constant temperature $T$. Nor can it be explained by the thermalization of the system itself on a timescale that is fast compared to slow energy dissipation by the bath, since the system is localized and nonergodic. Let us therefore investigate the underlying mechanism and its relation to Stark localization.

Underlying mechanism. Although a two-level system prepared in its excited state passes through the maximum entropy state while equilibrating with a thermal bath [76], such behavior is highly nontrivial for interacting many-body systems. To figure out the conditions for a close-to-maximum peak entropy, let us focus on the weak-coupling limit, where the secular approximation [37,38] gives a Lindblad master equation,

$$
\frac{d \rho}{d t}=-i[H, \rho]+\eta \sum_{k, q=1}^{M} R_{k q}\left(L_{k q} \rho L_{k q}^{\dagger}-\frac{1}{2}\left\{L_{k q}^{\dagger} L_{k q}, \rho\right\}\right),
$$

with rates $R_{k q} \equiv R_{k q q k}$. They obey $R_{k q} / R_{q k}=e^{-\left(\varepsilon_{k}-\varepsilon_{q}\right) / T}$, so that low-energy states are favored and the system is asymptotically driven towards the Gibbs state $\rho_{T}$. The matrix elements $\rho_{k q} \equiv\langle k|\rho| q\rangle$ follow $\dot{\rho}_{k q}=-i\left(\varepsilon_{k}-\varepsilon_{q}\right) \rho_{k q}+$
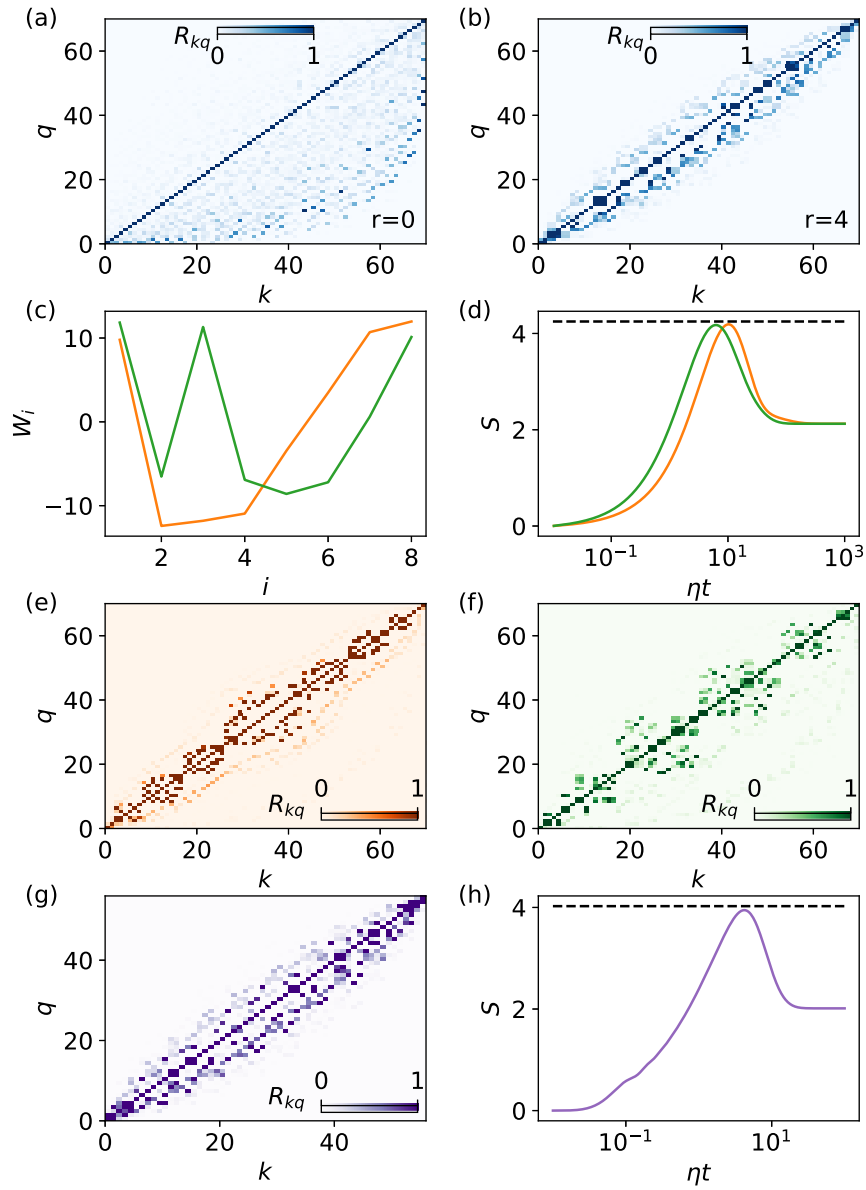

FIG. 2. (a), (b) Rate matrix for the Fermi-Hubbard chain coupled to a thermal bath, with (a) $r=0$ and (b) $r=4$. (c) Examples for on-site potentials $W_{i}$ that were optimized over the range $[-16,16]$ to provide a large peak entropy when evolving from the most excited state. The results are representative for 500 numerical runs. (d) Time evolution of the corresponding entropies. (e), (f) Corresponding rate matrices $R_{k q}$. (g) Rate matrix and (h) entropy for a tilted bosonic Hubbard chain with $r=4$ and initially all particles occupying the leftmost site. The system sizes are $M=8, N=4$ for (a)-(f), and $M=6, N=3$ for (g), (h). Other parameters are $V=1, \eta=0.1$, and $T$ so that $S_{T}=S_{\infty} / 2$.

$\eta \sum_{p}\left[R_{k p} \rho_{p p} \delta_{k q}-\frac{1}{2}\left(R_{p k}+R_{p q}\right) \rho_{k q}\right]$, where diagonal and off-diagonal elements decouple from each other. The latter decay with rates $\Gamma_{k q}=\frac{1}{2} \eta \sum_{p}\left(R_{p k}+R_{p q}\right)$ and have to be negligible already when the peak entropy is reached, to allow for the observed transient approach of the maximally mixed state (this is indeed the case-see Fig. S2 and discussion in Ref. [76]). The dynamics of the diagonal elements $p_{k} \equiv \rho_{k k}$ is determined by the rate matrix $R_{k q}$ through the Pauli rate equation $\dot{p}_{k}=\eta \sum_{q}\left[R_{k q} p_{q}-R_{q k} p_{k}\right]$ [37].

In Figs. 2(a) and 2(b) we compare rate matrices for $r=0$ and $r=4$. Without a potential gradient, one finds long-range coupling with respect to energy. In contrast, at a large field gradient $(r=4)$ the transition rates predominantly couple states that are close by in energy. The latter is a consequence of Stark localization, where eigenstates that are close in space, so that they are coupled by the bath via the densities $n_{i}$, are close also with respect to energy. Note that for a disorder- 
localized Fermi-Hubbard chain without this spatioenergetic correlation we find nonlocal rate matrices and no prethermal memory loss (see Fig. S10 of Ref. [76]). This constitutes an even more drastic difference between the relaxation dynamics of Stark and disorder-induced MBL than the one observed for dephasing noise [59].

To check whether a rate matrix with energy-local coupling is crucial for the appearance of a close-to-maximum entropy $\left(S_{\text {peak }} \simeq S_{\infty}\right)$, we investigate the rate matrices for rather different on-site potentials $W_{i}$ [see Fig. 2(c)] that (were optimized to) equally give rise to large peak entropies [see Fig. 2(d)]. It turns out that, indeed, they also show near-neighbor coupling [see Figs. 2(e) and 2(f)]. Moreover, also the tilted bosonic Hubbard chain [given by Eq. (1) with bosonic annihilation operators $c_{i}$ and the last term replaced by on-site interactions $\left.\frac{1}{2} V \sum_{i} n_{i}\left(n_{i}-1\right)\right]$ shows $S_{\text {peak }} \approx S_{\infty}$ together with an energy local rate matrix [see Figs. 2(g) and 2(h)]. This, together with results for simplified models shown below (and in Fig. S13 of Ref. [76]), indicates clearly that the prethermal memory loss discussed here is a very robust phenomenon.

Simplified rate model. To address the question why the appearance of a close-to-maximum entropy is associated with local coupling between energy states, let us now consider a simplified rate model. Here, the energy eigenstates have equally spaced nondegenerate energies $\varepsilon_{k}=r k$, with $k=$ $0,1, \ldots, \mathcal{D}-1$, and are coupled by thermal rates that are homogeneous and local with respect to energy. We define $R_{k+n, k} \equiv R_{n}$, where $R_{n}=0$ for $|n|>n_{m}$ and $R_{n}=g(n r)$ for $|n| \leqslant n_{m}$, so that (as a property of the bath correlation function g) $R_{n} / R_{-n}=\left(R_{+} / R_{-}\right)^{n}=e^{-n r / T}$, with $R_{ \pm} \equiv R_{ \pm 1}$.

Let us first study nearest-neighbor coupling, $n_{m}=1$. By defining $\nabla^{2} p_{k}=p_{k+1}+p_{k-1}-2 p_{k}$ and $\nabla p_{k}=\left(p_{k+1}-\right.$ $\left.p_{k-1}\right) / 2$, we can write the Pauli rate equation as a discrete drift-diffusion equation, $\dot{p}_{k}=\eta\left(\bar{R} \nabla^{2} p_{k}+\delta R \nabla p_{k}\right)$ [82], where diffusion and drift are quantified by $\bar{R} \equiv\left(R_{+}+R_{-}\right) / 2$ and $\delta R \equiv R_{-}-R_{+}$, respectively. Scaling time with $(\eta \bar{R})^{-1}$, the model is completely characterized by the ratio $\alpha=\delta R / \bar{R}$ and the system size $\mathcal{D}$. Starting from the highest excited state, $p_{k}(0)=\delta_{k, k_{\text {ini }}}$ with $k_{\text {ini }}=\mathcal{D}-1$, and setting $\mathcal{D}=50$, in Figs. 3(a) and 3(b) we plot $p_{k}$ for $\alpha=0.1$ and $\alpha=1$ for different times (solid lines, fat red lines indicate $t_{\text {peak }}$ ). While for $\alpha=0.1$, a rather uniform distribution is found at a time $t_{\text {peak }}$, approximating the maximum entropy state with $p_{k}=1 / \mathcal{D}$, this is not the case for larger drift, $\alpha=1$. Before reaching thermal equilibrium, we find the distribution well approximated by a Gaussian of standard deviation $\sigma=$ $\sqrt{2 \bar{R} t}$ centered at $k_{0}=k_{\text {ini }}-\delta R t$ [82] (dashed lines). The condition for reaching an almost flat distribution is thus given by the intuitive requirement that the drift time needed to reach $k_{0}=\mathcal{D} / 2, \tau_{F}=\left(k_{\text {ini }}-\mathcal{D} / 2\right) / \delta R$ is larger than the diffusion time giving rise to $\sigma=\mathcal{D} / 2, \tau_{D}=\mathcal{D}^{2} /(8 \bar{R})$. Thus, for $k_{\text {ini }}=$ $\mathcal{D}-1$ we expect $S_{\text {peak }} \approx S_{\infty}$ as long as $\alpha \lesssim 4 \mathcal{D}^{-1}$, which is confirmed in Fig. 3(c) [see also Fig. S12(a) of Ref. [76]].

However, $S_{\text {peak }} \approx S_{\infty}$ is a nontrivial result only as long as the thermal entropy $S_{T}$, plotted in Fig. 3(d), lies well below $S_{\infty}$. For $\mathcal{D} r \gg T$ we can neglect the upper bound of the spectrum and $S_{T}$ approaches the value for an harmonic oscillator with frequency $r$ [83], $S_{T} \simeq \frac{x}{x-1} \log (x)-\log (1-x)$ with $x=$ $e^{-r / T}=R_{+} / R_{-}=(2-\alpha) /(2+\alpha)$, so that for $\alpha \ll 1$ one
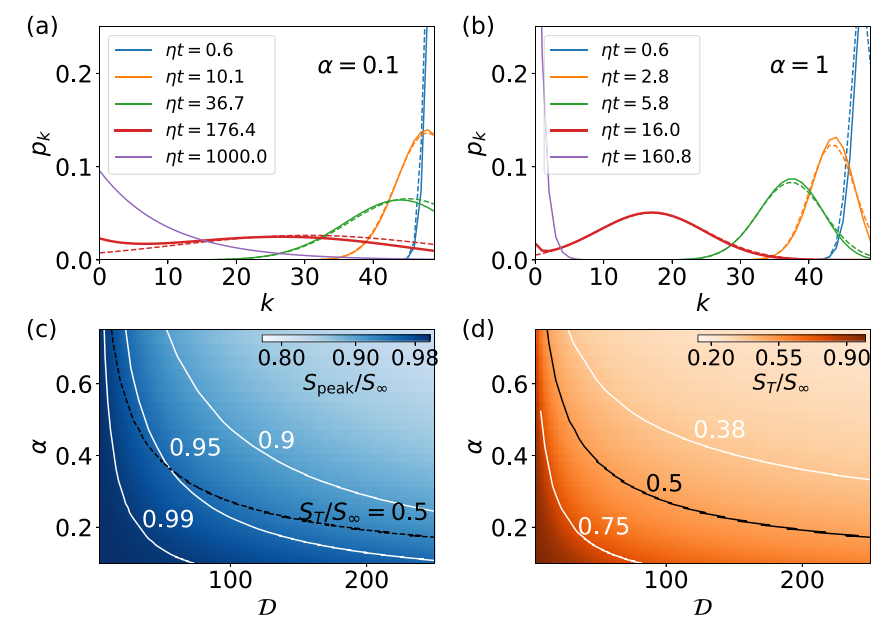

FIG. 3. Simplified rate model with nearest-neighbor coupling $n_{m}=1$ evolving from the most excited state. (a), (b) Probability distribution $p_{k}$ at different times for $\mathcal{D}=50$ and (a) $\alpha=0.1$ and (b) $\alpha=1$. (c), (d) Normalized peak and thermal entropies (c) $S_{\text {peak }} / S_{\infty}$ and (d) $S_{T} / S_{\infty}$ vs $\alpha$ and $\mathcal{D}$.

has $S_{T} \simeq \log (1 / \alpha)+1+\mathcal{O}(\alpha)$ [Fig. 3(d)]. Thus, $S_{T} / S_{\infty}<s$ as long as $\alpha \gtrsim e \mathcal{D}^{-s}$. While for $\mathcal{D} \rightarrow \infty$ this requirement is incompatible with the one for large peak entropies, $\alpha \lesssim 4 \mathcal{D}^{-1}$, it turns out that the different prefactors appearing in both conditions (whose values can deviate from our estimates $e$ and 4) still give rise to a large nontrivial regime for finite $\mathcal{D}$, as can be inferred from Figs. 3(c) and 3(d).

The simplified rate model with $n_{m}=1$ roughly corresponds to the case of a single particle in a tilted lattice. While it describes peak entropies $S_{\text {peak }} \approx S_{\infty}$ and prethermal memory loss, it gives rise to a Gaussian rather than an exponential prethermal distribution. This suggests that the formation of a prethermal Gibbs state requires more complex rate matrices as they are found for the many-particle case. In Fig. 4 we investigate what happens when increasing the coupling range $n_{m}$, and thus the complexity, of the simplified rate model (using $\mathcal{D}=50$ and $\eta=0.1$ ). We observe that $S_{\text {peak }}$ first increases with $n_{m}$ before, and after reaching a maximum at $n_{m}=11$, it decreases again. The first increase with $n_{m}$ might be explained by the prethermal distribution becoming more Gibbs-like and thus flatter at $t=t_{\text {peak }}$ than the Gaussian (which always retains a finite $\sigma$ ). This is confirmed in Fig. 4(b), where we plot the
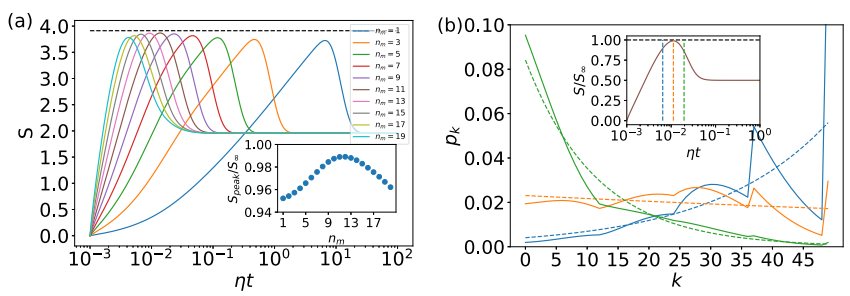

FIG. 4. Evolution of the simplified rate model starting from the most excited state for $\mathcal{D}=50, \eta=0.1$, and $T$ so that $S_{T}=S_{\infty} / 2$. (a) Entropy evolution for different $n_{m}$. Inset: Normalized peak entropies vs $n_{m}$. (b) Distribution $p_{k}$ (solid lines) for $n_{m}=11$ at the times marked in the inset compared to effective Gibbs states (dashed lines). 
distribution $p_{k}$ at different times for $n_{m}=11$ and find rather good agreement with an effective Gibbs state. The subsequent decrease of $S_{\text {peak }}$ can, in turn, be attributed to an increase of the drift velocity with $n_{m}$, which is clearly visible also in Fig. 4(a) and which reduces the time available for reaching a prethermal distribution. This mechanism explains why large transient peak entropies $S_{\text {peak }} \approx S_{\infty}$ are found for rate matrices that are local in energy.

Conclusion. We have shown that the nonequilibrium relaxation dynamics of interacting Wannier-Stark ladders coupled to a finite-temperature environment can feature effective prethermal memory loss. The effect is found to rely on a dissipative form of prethermalization. In an experiment with ultracold atoms, a thermal environment could be provided, for instance, by the coupling to a second atomic species [84].

Acknowledgments. We acknowledge discussions with Markus Oberthaler. This research was funded by the Deutsche Forschungsgemeinschaft (DFG, German Research Foundation) via the Research Unit FOR 2414 under Project No. 277974659.
[1] F. Bloch, Über die quantenmechanik der elektronen in kristallgittern, Z. Phys. 52, 555 (1929).

[2] G. H. Wannier, Wave functions and effective Hamiltonian for Bloch electrons in an electric field, Phys. Rev. 117, 432 (1960).

[3] C. Zener and R. H. Fowler, A theory of the electrical breakdown of solid dielectrics, Proc. R. Soc. London, Ser. A 145, 523 (1934).

[4] M. Glück, A. R. Kolovsky, and H. J. Korsch, Wannier-Stark resonances in optical and semiconductor superlattices, Phys. Rep. 366, 103 (2002).

[5] E. E. Mendez, F. Agulló-Rueda, and J. M. Hong, Stark Localization in GaAs-GaAlAs Superlattices under an Electric Field, Phys. Rev. Lett. 60, 2426 (1988).

[6] T. Pertsch, P. Dannberg, W. Elflein, A. Bräuer, and F. Lederer, Optical Bloch Oscillations in Temperature Tuned Waveguide Arrays, Phys. Rev. Lett. 83, 4752 (1999).

[7] R. Morandotti, U. Peschel, J. S. Aitchison, H. S. Eisenberg, and Y. Silberberg, Experimental Observation of Linear and Nonlinear Optical Bloch Oscillations, Phys. Rev. Lett. 83, 4756 (1999).

[8] S. R. Wilkinson, C. F. Bharucha, K. W. Madison, Q. Niu, and M. G. Raizen, Observation of Atomic Wannier-Stark Ladders in an Accelerating Optical Potential, Phys. Rev. Lett. 76, 4512 (1996).

[9] M. Ben Dahan, E. Peik, J. Reichel, Y. Castin, and C. Salomon, Bloch Oscillations of Atoms in an Optical Potential, Phys. Rev. Lett. 76, 4508 (1996).

[10] O. Morsch, J. H. Müller, M. Cristiani, D. Ciampini, and E. Arimondo, Bloch Oscillations and Mean-Field Effects of BoseEinstein Condensates in 1D Optical Lattices, Phys. Rev. Lett. 87, 140402 (2001).

[11] S. Kling, T. Salger, C. Grossert, and M. Weitz, Atomic BlochZener Oscillations and Stückelberg Interferometry in Optical Lattices, Phys. Rev. Lett. 105, 215301 (2010).

[12] H. Trompeter, W. Krolikowski, D. N. Neshev, A. S. Desyatnikov, A. A. Sukhorukov, Y. S. Kivshar, T. Pertsch, U. Peschel, and F. Lederer, Bloch Oscillations and Zener Tunneling in Two-Dimensional Photonic Lattices, Phys. Rev. Lett. 96, 053903 (2006).

[13] F. Dreisow, A. Szameit, M. Heinrich, T. Pertsch, S. Nolte, A. Tünnermann, and S. Longhi, Bloch-Zener Oscillations in Binary Superlattices, Phys. Rev. Lett. 102, 076802 (2009).

[14] S. Mukherjee, A. Spracklen, D. Choudhury, N. Goldman, P. Öhberg, E. Andersson, and R. R. Thomson, Modulationassisted tunneling in laser-fabricated photonic Wannier-Stark ladders, New J. Phys. 17, 115002 (2015).
[15] C. Schmidt, J. Bühler, A.-C. Heinrich, J. Allerbeck, R. Podzimski, D. Berghoff, T. Meier, W. G. Schmidt, C. Reichl, W. Wegscheider, D. Brida, and A. Leitenstorfer, Signatures of transient Wannier-Stark localization in bulk gallium arsenide, Nat. Commun. 9, 2890 (2018).

[16] E. Guardado-Sanchez, A. Morningstar, B. M. Spar, P. T. Brown, D. A. Huse, and W. S. Bakr, Subdiffusion and Heat Transport in a Tilted Two-Dimensional Fermi-Hubbard System, Phys. Rev. X 10, 011042 (2020).

[17] E. van Nieuwenburg, Y. Baum, and G. Refael, From Bloch oscillations to many-body localization in clean interacting systems, Proc. Natl. Acad. Sci. USA 116, 9269 (2019).

[18] M. Schulz, C. A. Hooley, R. Moessner, and F. Pollmann, Stark Many-Body Localization, Phys. Rev. Lett. 122, 040606 (2019).

[19] E. Altman and R. Vosk, Universal dynamics and renormalization in many-body-localized systems, Annu. Rev. Condens. Matter Phys. 6, 383 (2015).

[20] R. Nandkishore and D. A. Huse, Many-body localization and thermalization in quantum statistical mechanics, Annu. Rev. Condens. Matter Phys. 6, 15 (2015).

[21] F. Alet and N. Laflorencie, Many-body localization: An introduction and selected topics, C. R. Phys. 19, 498 (2018).

[22] D. A. Abanin, E. Altman, I. Bloch, and M. Serbyn, Colloquium: Many-body localization, thermalization, and entanglement, Rev. Mod. Phys. 91, 021001 (2019).

[23] V. Khemani, M. Hermele, and R. Nandkishore, Localization from Hilbert space shattering: From theory to physical realizations, Phys. Rev. B 101, 174204 (2020).

[24] R.-Y. Sun, Z. Zhu, and Z.-Yu. Weng, Localization in a $t-j$ Type Model with Translational Symmetry, Phys. Rev. Lett. 123, 016601 (2019).

[25] S. R. Taylor, M. Schulz, F. Pollmann, and R. Moessner, Experimental probes of Stark many-body localization, arXiv:1910.01154.

[26] M. Brenes, M. Dalmonte, M. Heyl, and A. Scardicchio, ManyBody Localization Dynamics from Gauge Invariance, Phys. Rev. Lett. 120, 030601 (2018).

[27] A. Smith, J. Knolle, R. Moessner, and D. L. Kovrizhin, Absence of Ergodicity without Quenched Disorder: From Quantum Disentangled Liquids to Many-Body Localization, Phys. Rev. Lett. 119, 176601 (2017).

[28] T. Grover and M. P. A. Fisher, Quantum disentangled liquids, J. Stat. Mech.: Theory Exp. (2014) P10010.

[29] M. Schiulaz, A. Silva, and M. Müller, Dynamics in many-body localized quantum systems without disorder, Phys. Rev. B 91, 184202 (2015). 
[30] N. Y. Yao, C. R. Laumann, J. I. Cirac, M. D. Lukin, and J. E. Moore, Quasi-Many-Body Localization in TranslationInvariant Systems, Phys. Rev. Lett. 117, 240601 (2016).

[31] Z. Papić, E. M. Stoudenmire, and D. A. Abanin, Many-body localization in disorder-free systems: The importance of finitesize constraints, Ann. Phys. 362, 714 (2015).

[32] A. Smith, J. Knolle, D. L. Kovrizhin, and R. Moessner, Disorder-Free Localization, Phys. Rev. Lett. 118, 266601 (2017).

[33] W. De Roeck and F. Huveneers, Asymptotic quantum manybody localization from thermal disorder, Commun. Math. Phys. 332, 1017 (2014).

[34] J. M. Hickey, S. Genway, and J. P. Garrahan, Signatures of many-body localisation in a system without disorder and the relation to a glass transition, J. Stat. Mech.: Theory Exp. (2016) 054047.

[35] M. van Horssen, E. Levi, and J. P. Garrahan, Dynamics of many-body localization in a translation-invariant quantum glass model, Phys. Rev. B 92, 100305(R) (2015).

[36] G. Carleo, F. Becca, M. Schiró, and M. Fabrizio, Localization and glassy dynamics of many-body quantum systems, Sci. Rep. 2, 243 (2012)

[37] H. P. Breuer and F. Petruccione, The Theory of Open Quantum Systems (Oxford University Press, Oxford, UK, 2002).

[38] H. J. Carmichael, Statistical Methods in Quantum Optics 1: Master Equations and Fokker-Planck Equations (Springer, Berlin, 2013).

[39] H. Pichler, A. J. Daley, and P. Zoller, Nonequilibrium dynamics of bosonic atoms in optical lattices: Decoherence of many-body states due to spontaneous emission, Phys. Rev. A 82, 063605 (2010).

[40] A. J. Daley, Quantum trajectories and open many-body quantum systems, Adv. Phys. 63, 77 (2014).

[41] J. P. Garrahan and I. Lesanovsky, Thermodynamics of Quantum Jump Trajectories, Phys. Rev. Lett. 104, 160601 (2010).

[42] S. Diehl, A. Micheli, A. Kantian, B. Kraus, H. P. Büchler, and P. Zoller, Quantum states and phases in driven open quantum systems with cold atoms, Nat. Phys. 4, 878 (2008).

[43] F. Verstraete, M. M. Wolf, and J. I. Cirac, Quantum computation and quantum-state engineering driven by dissipation, Nat. Phys. 5, 633 (2009).

[44] I. de Vega and D. Alonso, Dynamics of non-Markovian open quantum systems, Rev. Mod. Phys. 89, 015001 (2017).

[45] D. Vorberg, W. Wustmann, R. Ketzmerick, and A. Eckardt, Generalized Bose-Einstein Condensation into Multiple States in Driven-Dissipative Systems, Phys. Rev. Lett. 111, 240405 (2013)

[46] D. Vorberg, W. Wustmann, H. Schomerus, R. Ketzmerick, and A. Eckardt, Nonequilibrium steady states of ideal bosonic and fermionic quantum gases, Phys. Rev. E 92, 062119 (2015).

[47] A. Schnell, D. Vorberg, R. Ketzmerick, and A. Eckardt, HighTemperature Nonequilibrium Bose Condensation Induced by a Hot Needle, Phys. Rev. Lett. 119, 140602 (2017).

[48] D. Poletti, J.-S. Bernier, A. Georges, and C. Kollath, Interaction-Induced Impeding of Decoherence and Anomalous Diffusion, Phys. Rev. Lett. 109, 045302 (2012).

[49] J.-S. Bernier, R. Tan, L. Bonnes, C. Guo, D. Poletti, and C. Kollath, Light-Cone and Diffusive Propagation of Correlations in a Many-Body Dissipative System, Phys. Rev. Lett. 120, 020401 (2018).
[50] K. Baumann, C. Guerlin, F. Brennecke, and T. Esslinger, Dicke quantum phase transition with a superfluid gas in an optical cavity, Nature (London) 464, 1301 (2010).

[51] H. Ritsch, P. Domokos, F. Brennecke, and T. Esslinger, Cold atoms in cavity-generated dynamical optical potentials, Rev. Mod. Phys. 85, 553 (2013).

[52] M. Ludwig and F. Marquardt, Quantum Many-Body Dynamics in Optomechanical Arrays, Phys. Rev. Lett. 111, 073603 (2013).

[53] Y. Ashida, K. Saito, and M. Ueda, Thermalization and Heating Dynamics in Open Generic Many-Body Systems, Phys. Rev. Lett. 121, 170402 (2018).

[54] M. Nakagawa, N. Tsuji, N. Kawakami, and M. Ueda, Dynamical Sign Reversal of Magnetic Correlations in Dissipative Hubbard Models, Phys. Rev. Lett. 124, 147203 (2020).

[55] S. Deffner and E. Lutz, Nonequilibrium Entropy Production for Open Quantum Systems, Phys. Rev. Lett. 107, 140404 (2011).

[56] R. Labouvie, B. Santra, S. Heun, and H. Ott, Bistability in a Driven-Dissipative Superfluid, Phys. Rev. Lett. 116, 235302 (2016).

[57] H. P. Lüschen, P. Bordia, S. S. Hodgman, M. Schreiber, S. Sarkar, A. J. Daley, M. H. Fischer, E. Altman, I. Bloch, and U. Schneider, Signatures of Many-Body Localization in a Controlled Open Quantum System, Phys. Rev. X 7, 011034 (2017).

[58] L.-N. Wu, A. Schnell, G. De Tomasi, M. Heyl, and A. Eckardt, Describing many-body localized systems in thermal environments, New J. Phys. 21, 063026 (2019).

[59] L.-N. Wu and A. Eckardt, Bath-Induced Decay of Stark ManyBody Localization, Phys. Rev. Lett. 123, 030602 (2019).

[60] E. Levi, M. Heyl, I. Lesanovsky, and J. P. Garrahan, Robustness of Many-Body Localization in the Presence of Dissipation, Phys. Rev. Lett. 116, 237203 (2016).

[61] M. H. Fischer, M. Maksymenko, and E. Altman, Dynamics of a Many-Body-Localized System Coupled to a Bath, Phys. Rev. Lett. 116, 160401 (2016).

[62] M. V. Medvedyeva, T. Prosen, and M. Žnidarič, Influence of dephasing on many-body localization, Phys. Rev. B 93, 094205 (2016).

[63] B. Everest, I. Lesanovsky, J. P. Garrahan, and E. Levi, Role of interactions in a dissipative many-body localized system, Phys. Rev. B 95, 024310 (2017).

[64] M. Žnidarič, J. J. Mendoza-Arenas, S. R. Clark, and J. Goold, Dephasing enhanced spin transport in the ergodic phase of a many-body localizable system, Ann. Phys. 529, 1600298 (2016).

[65] F. Nissen, S. Schmidt, M. Biondi, G. Blatter, H. E. Türeci, and J. Keeling, Nonequilibrium Dynamics of Coupled Qubit-Cavity Arrays, Phys. Rev. Lett. 108, 233603 (2012).

[66] M. Marcuzzi, E. Levi, S. Diehl, J. P. Garrahan, and I. Lesanovsky, Universal Nonequilibrium Properties of Dissipative Rydberg Gases, Phys. Rev. Lett. 113, 210401 (2014).

[67] D. Tamascelli, A. Smirne, J. Lim, S. F. Huelga, and M. B Plenio, Efficient Simulation of Finite-Temperature Open Quantum Systems, Phys. Rev. Lett. 123, 090402 (2019).

[68] X. Xu, J. Thingna, C. Guo, and D. Poletti, Many-body open quantum systems beyond Lindblad master equations, Phys. Rev. A 99, 012106 (2019).

[69] J.-S. Wang, B. K. Agarwalla, H. Li, and J. Thingna, Nonequilibrium Green's function method for quantum thermal transport, Front. Phys. 9, 673 (2014). 
[70] C. Gardiner, P. Zoller, and P. Zoller, Quantum Noise: A Handbook of Markovian and non-Markovian Quantum Stochastic Methods with Applications to Quantum Optics, Vol. 56 (Springer, Berlin, 2004).

[71] C. Guo, I. de Vega, U. Schollwöck, and D. Poletti, Stableunstable transition for a Bose-Hubbard chain coupled to an environment, Phys. Rev. A 97, 053610 (2018).

[72] R. Tan, X. Xu, and D. Poletti, Interaction-impeded relaxation in the presence of finite-temperature baths, Phys. Rev. A 101, 023603 (2020).

[73] M. Prüfer, P. Kunkel, H. Strobel, S. Lannig, D. Linnemann, C.-M. Schmied, J. Berges, T. Gasenzer, and M. K. Oberthaler, Observation of universal dynamics in a spinor Bose gas far from equilibrium, Nature (London) 563, 217 (2018).

[74] S. Erne, R. Bücker, T. Gasenzer, J. Berges, and J. Schmiedmayer, Universal dynamics in an isolated onedimensional Bose gas far from equilibrium, Nature (London) 563, 225 (2018).

[75] P. Jordan and E. Wigner, Über das paulische äquivalenzverbot, Z. Phys. 47, 631 (1928).

[76] See Supplemental Material at http://link.aps.org/supplemental/ 10.1103/PhysRevB.101.220302 for the discussion of a twolevel system, dependence of entropy for the Stark model on bath temperature and initial state, more details on the universal behavior of mean occupation in real space for the Stark model, the rate matrix and entropy of the disordered-potential model, and more details on the simplified rate model.

[77] "Effective," since formally the initial state can still be recovered by evolving backwards in time.

[78] J. Berges, A. Rothkopf, and J. Schmidt, Nonthermal Fixed Points: Effective Weak Coupling for Strongly Correlated Systems Far from Equilibrium, Phys. Rev. Lett. 101, 041603 (2008).

[79] A. Piñeiro Orioli, K. Boguslavski, and J. Berges, Universal selfsimilar dynamics of relativistic and nonrelativistic field theories near nonthermal fixed points, Phys. Rev. D 92, 025041 (2015).

[80] B. Nowak, D. Sexty, and T. Gasenzer, Superfluid turbulence: Nonthermal fixed point in an ultracold Bose gas, Phys. Rev. B 84, 020506(R) (2011).

[81] J. Berges, S. Borsányi, and C. Wetterich, Prethermalization, Phys. Rev. Lett. 93, 142002 (2004).

[82] S. Chandrasekhar, Stochastic problems in physics and astronomy, Rev. Mod. Phys. 15, 1 (1943).

[83] H. Gould and J. Tobochnik, Statistical and Thermal Physics: With Computer Applications (Princeton University Press, Princeton, NJ, 2010).

[84] F. Schmidt, D. Mayer, Q. Bouton, D. Adam, T. Lausch, N. Spethmann, and A. Widera, Quantum Spin Dynamics of Individual Neutral Impurities Coupled to a Bose-Einstein Condensate, Phys. Rev. Lett. 121, 130403 (2018). 